\title{
Mensuração da Pressão Intra-Abdominal nas Unidades de Tratamento Intensivo. A Opinião dos Médicos Intensivistas*
}

\author{
Measurement of Intra-Abdominal Pressure in the Intensive \\ Care Unit. The Opinion of the Critical Care Physicians
}

\author{
André M. Japiassú1, Haroldo Falcão ${ }^{1,2}$, Fernando Freitas ${ }^{3,7}$, Sandra Freitas ${ }^{1,4}$, Paulo César P. Souza ${ }^{5,6,7}$, \\ Roberto Lannes ${ }^{1}$, Ricardo I. Sato ${ }^{7}$, Analucia M. Dias ${ }^{3,7}$, Gustavo F. Almeida ${ }^{8}$, Márcio Soares ${ }^{9}$, Jorge I. Salluh ${ }^{9,10}$
}

\section{RESUMO}

JUSTIFICATIVA E OBJETIVOS: Os efeitos adversos da hipertensão intra-abdominal (PIA) são conhecidos há muitos anos, mas apenas recentemente deu-se importância à sua monitorização. Há evidências que cerca de um quarto dos centros de tratamento intensivo (CTI) não medem a PIA por falta de conhecimento da sua importância ou dificuldade na interpretação dos resultados. O objetivo deste estudo foi avaliar o conhecimento dos médicos sobre a síndrome de compartimento abdominal (SCA) e as características do seu manuseio.

MÉTODO: Para a realização deste estudo foi enviado um questionário, contendo 12 perguntas sobre o assunto, para médicos que trabalham em CTI.

RESULTADOS: O conhecimento das definições internacionais de SCA não parece estar influenciado pelo tempo de exercício da Medicina, mas sim pelo tempo

1. Hospital Quinta D'Or, Rio de Janeiro, RJ

2. COTEFIL Hospital de Clínicas, Duque de Caxias, RJ

3. Hospital Universitário Clementino Fraga Filho, Rio de Janeiro, RJ

4. Hospital Vital, Rio de Janeiro, RJ

5. Hospital de Clínicas de Niterói, Niterói, RJ

6. Hospital de Clínicas Mario Lioni, Duque de Caxias, RJ

7. Hospital Evangélico, Rio de Janeiro, RJ

8. Casa de Saúde São José, Rio de Janeiro, RJ

9. Instituto Nacional do Câncer, Rio de Janeiro, RJ

10. Hospital Barra D'Or, Rio de Janeiro, RJ

*Recebido do Hospital Quinta D’Or, Rio de Janeiro, RJ

Apresentado em 26 de janeiro de 2007

Aceito para publicação em 27 de março de 2007

Endereço para correspondência

Dr. André M Japiassú

Rua Almirante Baltazar 435 - São Cristóvão

20941-150 Rio de Janeiro, RJ

E-mail: andrejapi@gmail.com

(C)Associação de Medicina Intensiva Brasileira, 2007 de atividade dedicada à Medicina Intensiva. Embora a maioria esteja ciente da existência da SCA, menos da metade dos médicos que responderam ao questionário conhece as definições internacionais de 2004. A medida da PIA é realizada em pacientes com predisposição para SCA, por via intravesical, com injeção de 25 a $100 \mathrm{~mL}$ de líquido, com intervalos de 4 a 8 horas. Não parece existir valor de PIA (associado ou não a disfunções orgânicas) de consenso entre médicos desta pesquisa em relação ao tratamento clínico ou cirúrgico.

CONCLUSÕES: O conhecimento sobre SCA é satisfatório quando considerados apenas os médicos que atuam majoritariamente em Medicina Intensiva. Contudo, é necessária a educação acerca da presença e gravidade da hipertensão intra-abdominal para grande parte dos médicos atuantes na Medicina Intensiva na região metropolitana do Rio de Janeiro.

Unitermos: laparotomia descompressiva, Medicina intensiva, pressão vesical, pressão intra-abdominal, síndrome de compartimento abdominal.

\section{SUMMARY}

BACKGROUND AND OBJECTIVES: The adverse effects of intra-abdominal hypertension are known for many years. Only recently proper attention has been given to routine intra-abdominal pressure (IAP) monitoring. There is evidence that a quarter of intensive care units (ICU) do not measure IAP, due to a lack of knowledge of its importance or difficulty in results interpretation. The aim of this study is investigate the knowledge of ICU physicians about abdominal compartimental syndrome and its management.

METHODS: A questionnaire with 12 questions about this issue was mailed to ICU physicians.

RESULTS: The current knowledge of the international definitions of ACS does not seem to be linked to the 
number of years of medical practice, but was associated with the time spent working on intensive care. Although most physicians are aware of the existence of ACS, less than half know the present international definitions. The IAP monitoring is performed in patients at risk for ACS, by means of the intravesical filling with 25 to $100 \mathrm{~mL}$ of liquids, in intervals varying from of 4 to 8 hours. There was no consensus on the value of IAP values (with or without organ dysfunctions) for the clinical or surgical treatments of ACS in this survey.

CONCLUSIONS: The knowledge of ACS is satisfactory when we consider only physicians that devote most of their time to ICU work. However, it is necessary to improve education and knowledge of most intensive care physicians regarding the presence and severity of intraabdominal hypertension in Rio de Janeiro.

Key Words: abdominal compartment syndrome, bladder pressure, critical care, decompression laparotomy, intra-abdominal pressure.

\section{INTRODUÇÃO}

Os efeitos adversos da hipertensão intra-abdominal são conhecidos há muitos anos ${ }^{1,2}$, mas apenas recentemente deu-se importância clínica à pressão intra-abdominal (PIA) elevada. Kron e col. foram os pioneiros na medida de PIA em pacientes cirúrgicos e a usavam para indicar laparotomia descompressiva ${ }^{3}$. Desde então, os efeitos da laparotomia na síndrome de compartimento abdominal (SCA) são valorizados e melhor reconhecidos. A PIA elevada foi associada com desenvolvimento de síndrome de disfunção orgânica múltipla (SDOM) e alta letalidade 4 .

A PIA normal varia entre 0 e $12 \mathrm{mmHg}$ e pode estar relacionada ao índice de massa corporal (IMC) de acordo com alguns autores ${ }^{5,6}$. Pressões acima de 15 a 20 mmHg são capazes de causar redução do débito urinário, aumento da pressão respiratória e redução do débito cardíaco. Quando maiores que 25 $\mathrm{mmHg}$, mudanças fisiológicas são freqüentes e clinicamente significativas ${ }^{6}$ : muitos médicos consideram este nível de PIA suficiente para indicar descompressão cirúrgica.

A incidência e a prevalência de hipertensão intra-abdominal (HIA) são elevadas e dependem da natureza da população estudada ${ }^{7,8}$, mas poucos centros têm uma rotina de medida de PIA estabelecida. Em grande parte dos casos ela é medida na suspeita clínica de hipertensão intra-abdominal. Recentemente, a PIA tem sido utilizada conjuntamente com medidas respiratórias para ajuste da ventilação mecânica? .

Embora o número de publicações sobre a síndrome compartimental abdominal tenha crescido de modo exponencial nos últimos 15 anos, incertezas permanecem sobre seu diagnóstico e tratamento. Estudo recente mostra uma freqüência relativamente baixa de medidas de PIA em hospitais ingleses ${ }^{10}$. Cerca de um quarto dos centros de tratamento intensivo não medem PIA por falta de conhecimento da sua importância ou dificuldade de interpretação dos resultados.

Após o Congresso Mundial sobre o tema em 2004, a HIA foi definida como a presença repetida ou sustentada de PIA $\geq 12 \mathrm{mmHg}$. A SCA ocorre nos casos de PIA > $20 \mathrm{mmHg}$, na presença de pelo menos uma nova disfunção orgânica ${ }^{11}$.

O objetivo deste estudo foi avaliar o conhecimento dos médicos sobre a síndrome de hipertensão intra-abdominal e as características do seu manuseio.

\section{MÉTODO}

Foi elaborado um questionário, submetido ao Comitê de Ética da Rede Lab's D'Or e aprovado em julho de 2006. Ele continha 12 perguntas e foi enviado por correio eletrônico a 153 médicos que trabalham em centros de tratamento intensivo de 10 hospitais do Rio de Janeiro, Duque de Caxias e Niterói. Essas unidades têm características clínico-cirúrgicas, reúnem 258 leitos e internam cerca de 8.000 pacientes por ano. Não foi necessário identificar-se ou citar a instituição.

O questionário incluía questões sobre a experiência profissional (tempo de atividade em Medicina Intensiva e de experiência após a graduação). A experiência profissional foi medida em intervalos de 5 anos: 1-5, 6-10, $11-15$ e mais de 16 anos de graduação. Os profissionais foram divididos de acordo com o tempo semanal dedicado à Medicina Intensiva: "pequeno", se menor que $25 \%$, "moderado" se entre $26 \%$ e $50 \%$, "grande", se entre $51 \%$ e $75 \%$ e "intenso" se maior que $76 \%$. O questionário está demonstrado no anexo 1.

Os dados considerados foram: conhecimento da síndrome de compartimento abdominal; experiência com medida prática de PIA; via de mensuração de PIA (vesical, gástrica, femoral ou outra); volume de líquido administrado pelo cateter vesical ou gástrico antes da medida da PIA; indicações de monitorização de PIA (laparotomia de urgência, reposição volêmica maciça, ventilação mecânica, lesão pulmonar aguda, suspeita clínica de SCA); freqüência de medida de PIA; 
nível de PIA capaz de indicar tratamento clínico; nível de PIA capaz de indicar tratamento cirúrgico (laparotomia descompressiva); conhecimento das definições da Sociedade Mundial de Síndrome de Compartimento Abdominal (WSACS) ${ }^{11}$.

As respostas foram avaliadas descritiva e comparativamente de acordo com o grau de experiência, tempo de atividade semanal dedicado à Medicina intensiva e conhecimento das definições de HIA/SCA da WSACS.

\section{RESULTADOS}

O retorno foi de 90 questionários, ou seja $58,8 \%$ de adesão. Sessenta e oito por cento dos médicos que responderam ao questionário têm entre 1 e 10 anos de experiência após a graduação e $64 \%$ têm pelo menos metade de seu tempo dedicado à Medicina Intensiva (Tabela 1). A maioria está ciente da existência da síndrome de compartimento abdominal e utiliza a medida de PIA (92\%).

Tabela 1 - Características dos Médicos Participantes

\begin{tabular}{lcc}
\hline Questão & Respostas & $\begin{array}{c}\text { Número de } \\
\text { Respostas (\%)*\# }\end{array}$ \\
\hline Quantos anos de experiên- & $1-5$ anos & $38(42 \%)$ \\
cia têm após a graduação & $6-10$ anos & $23(26 \%)$ \\
em Medicina? & $11-15$ anos & $12(13 \%)$ \\
& $>16$ anos & $17(19 \%)$ \\
Qual é o tempo de ativi- & $<25 \%$ & $12(13 \%)$ \\
dade dedicada à Medicina & $26 \%-50 \%$ & $20(22 \%)$ \\
Intensiva? & $51 \%-75 \%$ & $20(22 \%)$ \\
& $>76 \%$ & $38(43 \%)$ \\
Qual é o método que você & Intravesical & $81(97 \%)$ \\
emprega para medir a pres- & Gástrica & $1(1,2 \%)$ \\
são intra-abdominal? & Femoral & $2(2,5 \%)$ \\
& Outra & $1(1,2 \%)$ \\
Qual volume de líquido é & $0-20 \mathrm{~mL}$ & $7(8,4 \%)$ \\
administrado antes da me- & $25-50 \mathrm{~mL}$ & $31(37,3 \%)$ \\
dida da pressão na cavida- & $60-100 \mathrm{~mL}$ & $45(54,3 \%)$ \\
de vesical (outra)? & $>110 \mathrm{~mL}$ & $0(0 \%)$ \\
& & $12(14,8 \%)$ \\
Qual é a freqüência da & $0-4 \mathrm{~h}$ & $50(60 \%)$ \\
medida de pressão intra- & $4-8 \mathrm{~h}$ & $13(15,5 \%)$ \\
abdominal que você utiliza & $12 \mathrm{~h}$ & $7(8,5 \%)$ \\
como rotina? & $24 \mathrm{~h}$ & \\
& a critério clínico & \\
\hline
\end{tabular}

*Dados descritos em números absolutos e porcentagem

"Porcentagem calculada baseada no número de respostas de cada questão

Quase todos medem a PIA por via intravesical (97\%). Apenas a minoria mede por outras vias, como femoral ou gástrica.

Existe, porém, controvérsia em relação ao volume a ser administrado antes da medida da PIA, pelo método intravesical. $\mathrm{O}$ grupo parece dividido entre usar volumes de 25 a $50 \mathrm{~mL}(37,3 \%)$ e de 60 a $100 \mathrm{~mL}(54,3 \%)$, sendo que poucos profissionais preferem pequenos volumes de até $20 \mathrm{~mL}(8,4 \%)$.

Mais heterogênea ainda é a freqüência com a qual a medida de PIA é empregada: intervalos de 4, 8, 12 ou 24 horas e até mesmo apenas a critério clínico, ou seja, sem periodicidade definida. Deve-se destacar que o intervalo de 4 a 8 horas, que é a preferência de $60 \%$ dos médicos.

A predisposição clínica para desenvolver SCA é a maior indicação para aferir PIA $(51,4 \%)$. Outras indicações para monitorização da PIA têm as seguintes preferências: laparotomia de urgência (18\%); síndrome de angústia respiratória aguda (16,2\%); reposição volêmica maciça (13,5\%); e pacientes em ventilação mecânica invasiva (0,9\%) (Figura 1).

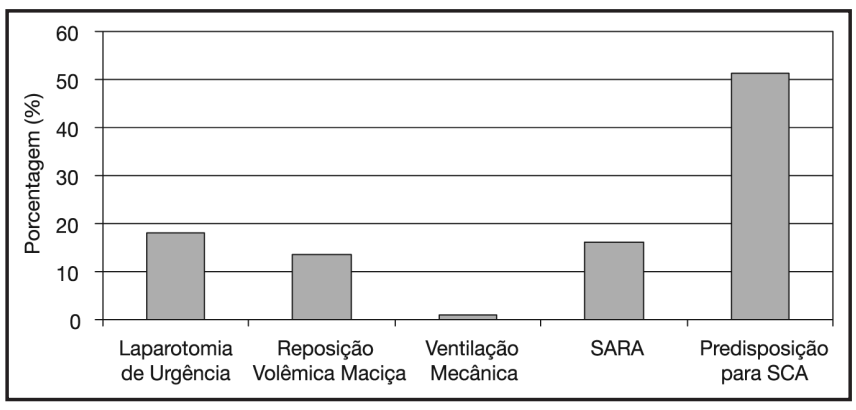

Figura 1 - Indicações para Monitorização de Pressão Intra-Abdominal

Embora a recomendação do consenso sobre HIA/SCA sugira tratar clinicamente a PIA acima de $12 \mathrm{mmHg}$, apenas $45 \%$ seguiram esta orientação (Figura 2). Grande parte (28\%) prefere analisar outros aspectos clínicos junto com a medida de PIA a fim de iniciar o tratamento. A indicação de laparotomia, ou seja, tratamento cirúrgico, também foi discordante entre os respondedores (Figura 3). A presença de disfunções orgânicas concomitantes nem sempre é valorizada; e, para muitos médicos, a realização de laparotomia se define com níveis de PIA de 20, 25 ou $30 \mathrm{mmHg}$, isoladamente. A opção mais freqüentemente escolhida foi PIA de $20 \mathrm{mmHg}$, não importando a presença de disfunções orgânicas (31\%). Neste contexto, é válido lembrar que o consenso aponta a presença de SCA com PIA maior que $20 \mathrm{mmHg}$, associada a novas disfunções orgânicas o que foi concordante com $12 \%$ das respostas. 


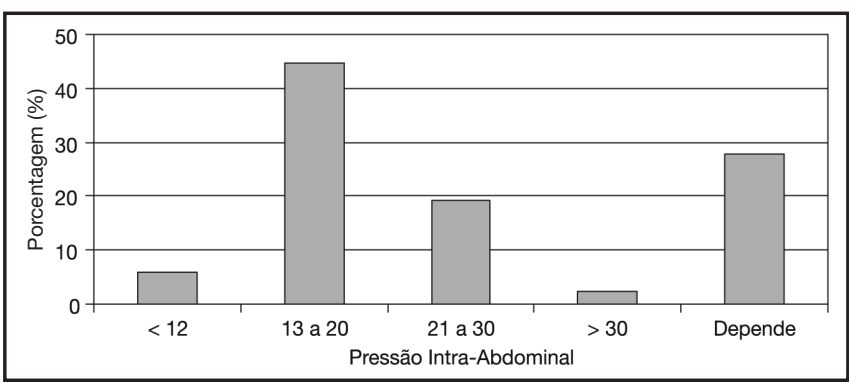

Figura 2 - Pressão Intra-Abdominal Crítica para Iniciar Tratamento Clínico

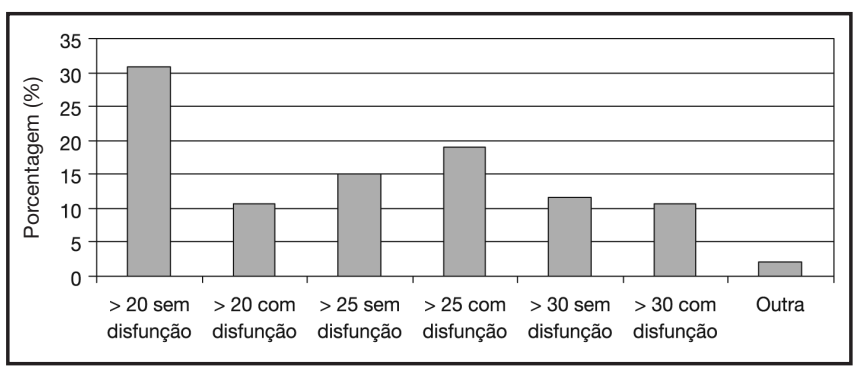

Figura 3 - Pressão Intra-Abdominal Crítica para Iniciar Tratamento Cirúrgico (Laparotomia)

Analisou-se também se algumas respostas tiveram influência dessas definições e recomendações da WSACS. Trinta e quatro médicos (38\%) declararam conhecer as definições de hipertensão intra-abdominal da WSACS. O tempo de exercício da Medicina não parece influenciar o conhecimento das definições (55\% versus $67 \%$ ). Entretanto, se o tempo de dedicação à Medicina Intensiva é maior que $51 \%$, observa-se com maior freqüência o conhecimento das definições internacionais de HIA SCA (dedicação grande/intensa $75 \%$ versus dedicação pequena/moderada $24 \%$ ). Não foi encontrada associação entre as indicações de tratamento clínico ou cirúrgico e o conhecimento destas definições (Figuras 4 e 5).

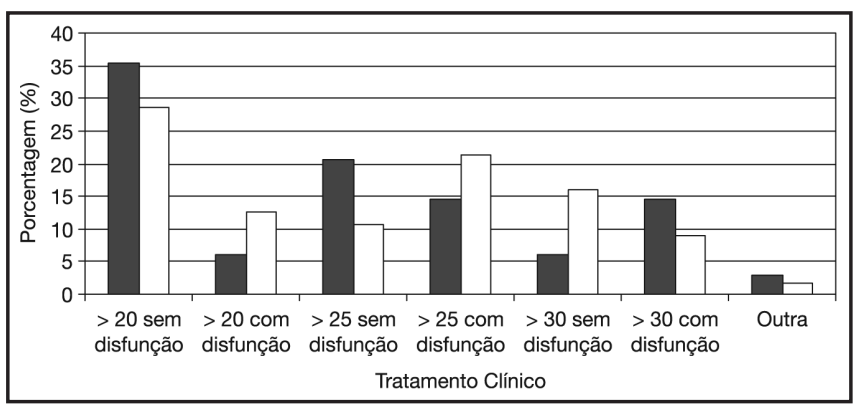

Figura 4 - Conhecimento das Definições da WSACS e Influência na Pressão Intra-Abdominal para Tratamento Clínico.

As colunas pretas correspondem aos médicos que conhecem as definições da WSACS e as colunas brancas correspondem aos médicos que não as conhecem. Não houve diferença significativa entre o conhecimento ou não das definições, entre os diferentes níveis de PIA e a presença de disfunções orgânicas.

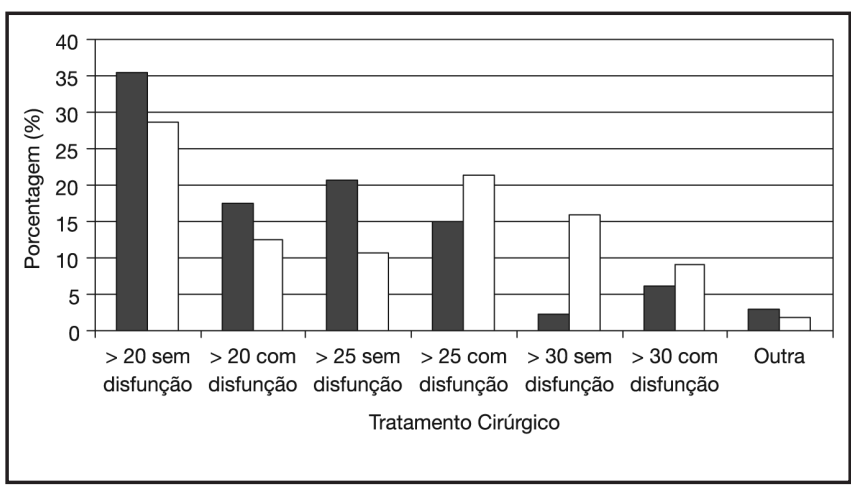

Figura 5 - Conhecimento das Definições da WSACS e Influência na Pressão Intra-Abdominal para Tratamento Cirúrgico.

As colunas pretas correspondem aos médicos que conhecem as definições da WSACS e as colunas brancas correspondem aos médicos que não as conhecem. Não houve diferença significativa entre o conhecimento ou não das definições, entre os diferentes níveis de PIA e a presença de disfunções orgânicas.

\section{DISCUSSÃO}

As observações deste estudo são semelhantes aos resultados de pesquisas realizadas nos Estados Unidos e Inglaterra nos últimos 2 anos ${ }^{10,12}$. Apesar do conhecimento da síndrome, não existe consenso na freqüência ou na indicação para medida de PIA, assim como nos tratamentos clínico e cirúrgico. No presente estudo repete-se o resultado do estudo inglês, no qual grande parte dos médicos prefere monitorizar a PIA em casos de risco para $\mathrm{SCA}^{8}$. A consideração de risco, cujo conteúdo é subjetivo e por isso variável entre os profissionais, parece ser um importante determinante no processo de instituição da medida da PIA, muito mais que a presença de indicações formais e objetivas.

Além das dúvidas acerca das indicações de medida da PIA, outro ponto problemático sobre a metodologia da medida surgiu a partir dos questionários respondidos: o valor de líquido instilado. Recente estudo clínico demonstrou a suficiência de pequenos volumes (até 25 $\mathrm{mL}$ ) para medida confiável da PIA. Mesmo alíquotas tão pequenas seriam capazes de eliminar o ar do sistema ${ }^{13}$. Volumes maiores que $50 \mathrm{~mL}$ se associaram a valores superestimados de pressão, sendo que a instilação de 300 $\mathrm{mL}$, por exemplo, definia HIA em $93 \%$ dos pacientes e SCA em 52\%. Nos questionários respondidos, observou-se que mais da metade dos profissionais utilizam volumes maiores que $60 \mathrm{~mL}$, o que pode superestimar a incidência do distúrbio.

Tendo em vista o exposto, o conhecimento das definições da WSACS não parece estar influenciado pelo tempo de exercício de profissão dos médicos que atuam nesta especialidade, mas sim pelo tempo 
de atividade a ela dedicada. Embora a maioria esteja ciente da existência da HIA/SCA, menos da metade conhece as definições da WSACS de 2004. A medida da PIA é realizada em pacientes com predisposição a SCA, por via intravesical, com injeção de 25 a $100 \mathrm{~mL}$ de líquido, com intervalos de 4 a 8 horas. Não parece existir um valor de PIA (associado ou não a disfunções orgânicas) de consenso entre os médicos desta pesquisa que indique tratamento clínico ou cirúrgico da HIA/SCA.
Ainda que de escopo limitado, os resultados dos questionários dão margem a importantes questionamentos sobre a heterogeneidade do conhecimento sobre a síndrome compartimental abdominal neste estado. A premência de uma investigação mais abrangente faz-se ainda mais ressaltada se for considerada a importância dos hospitais nesta avaliação preliminar. Será necessário ainda investigar se o conhecimento do consenso da WSACS é um facilitador para a aplicação das mensurações das pressões intra-abdominais em nosso meio.

Anexo 1 - Questionário sobre a Opinião sobre a Mensuração da Pressão Intra-Abdominal no CTI

1. Qual é o tempo de atividade dedicada à Medicina Intensiva ?

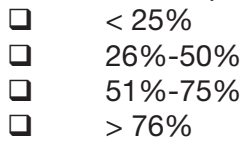

2. Quantos anos de experiência após graduação em Medicina ?

口 1-5 anos

․ 6-10 anos

ㅁ 11-15 anos

ㄱ $>16$ anos

3. Você está ciente do conceito de síndrome de compartimento abdominal ?

$\begin{array}{ll}\square & \text { SIM } \\ \square & \text { NÃO }\end{array}$

4. Você já mediu a pressão intra-abdominal em algum dos seus pacientes ?

口 SIM

NÃO

(Se SIM, siga para a pergunta 5; se NÃO, siga para a pergunta 11)

5. Qual é o método que você emprega para medir pressão intra-abdominal ?

a Intravesical

G Gástrica

口 Femoral

O Outra (especifique):

6. Qual é o volume de líquido que você geralmente pede para ser injetado pelo cateter vesical ou gástrico, antes da medida da pressão intravesical ?

口 $\quad 0-20 \mathrm{~mL}$

$25-50 \mathrm{~mL}$

60-100 mL

$>110 \mathrm{~mL}$

7. Em que tipos de pacientes indicaria a mensuração de pressão intra-abdominal?

- Todos os pacientes cirúrgicos internados no CTI após laparotomia de urgência

Após reposição volêmica maciça ( $p$. ex.: choque séptico)

Qualquer paciente em ventilação mecânica invasiva

Ventilação mecânica e SARA (relação $\mathrm{PaO}_{2} / \mathrm{FiO}_{2}<200$ )

Somente em pacientes predispostos a desenvolver síndrome de compartimento abdominal

8. Qual é a freqüência da medida de pressão intra-abdominal que você utiliza como rotina?

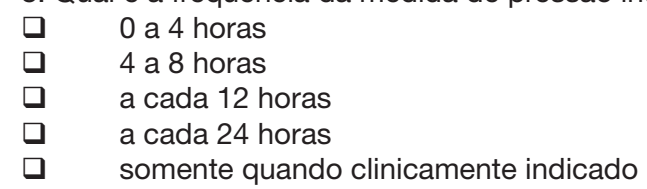


9. Quando você recomendaria tratamento clínico?

$\mathrm{PIA}>12 \mathrm{mmHg}$

PIA 13-20 mmHg

PIA $21-30 \mathrm{mmHg}$

$\mathrm{PIA}>31 \mathrm{mmHg}$

Depende dos sinais clínicos do paciente

10. Quando você recomendaria descompressão cirúrgica (laparotomia)?

PIA persistentemente acima de $30 \mathrm{mmHg}$, não importando os sinais de disfunção orgânica

PIA persistentemente acima de $30 \mathrm{mmHg}$, com sinais de uma ou mais disfunções orgânicas

PIA persistentemente acima de $25 \mathrm{mmHg}$, não importando os sinais de disfunção orgânica

PIA persistentemente acima de $25 \mathrm{mmHg}$, com sinais de uma ou mais disfunções orgânicas

PIA persistentemente acima de $20 \mathrm{mmHg}$, não importando os sinais de disfunção orgânica

PIA persistentemente acima de $20 \mathrm{mmHg}$, com sinais de uma ou mais disfunções orgânicas

Você indicaria outro valor limítrofe de PIA, indique: $\mathrm{mmHg}$

11. Se você NÃO mede pressão intra-abdominal, é porque: (pode marcar 1 ou mais opções)

口 Não sabe como medir

Sente que é perda de tempo

Não sabe como interpretar os resultados da medida

Nunca admitiu pacientes com hipertensão intra-abdominal

12. Você conhece as definições do consenso da Sociedade Mundial de Síndrome de Compartimento Abdominal ?

SIM

NÃO

\section{REFERÊNCIAS}

01. Emerson H - Intra-abdominal pressures. Arch Intern Med, 1911;7:754784.

02. Bradley SE, Bradley GP - The effect of increased abdominal pressure on renal function. J Clin Invest, 1947;26:1010-1015.

03. Kron IL, Harman PK, Nolan SP - Measurement of intra-abdominal pressure as a criterion for abdominal re-exploration. Ann Surg, 1984;199:28-30.

04. Balogh Z, McKinley BA, Holcomb JB et al - Both primary and secondary abdominal compartment syndrome can be predicted early and are harbingers of multiple organ failure. J Trauma, 2003;54:848-861.

05. Malbrain ML, Deeren D, De Potter TJ - Intra-abdominal hypertension in the critically ill: it is time to pay attention". Curr Op Crit Care, 2005;11:156-171.

06. Hunter JD, Damani Z - Intra-abdominal hypertension and the abdominal compartment syndrome. Anaesthesia, 2004;59:899-907.

07. Malbrain ML, Chiumello D, Pelosi P et al - Prevalence of intra-abdominal hypertension in critically ill patients: a multicentre epidemiological study. Intensive Care Med, 2004;30:822-829.
08. Malbrain ML, Chiumello D, Pelosi P et al - Incidence and prognosis of intraabdominal hypertension in a mixed population of critically ill patients: a multiple-center epidemiological study. Crit Care Med, 2005;33:315-322.

09. Rouby JJ, Puybasset L, Nieszkowska A et al - Acute respiratory distress syndrome: lessons from computed tomography of the whole lung. Crit Care Med, 2003;31:(Suppl4):S285-S295.

10. Ravishankar N, Hunter $\mathrm{J}$ - Measurement of intra-abdominal pressure in intensive care units in the United Kingdom: a national postal questionnaire study. Br J Anaesth, 2005;94:763-766.

11. Disponível em http://www.wsacs.org/consensus_definitions.htm Acessado em 8 de janeiro de 2007.

12. Kimball EJ, Rollins MD, Mone MC et al - Survey of intensive care unit physicians on the recognition and management of intra-abdominal hypertension and abdominal compartment syndrome. Crit Care Med, 2006;34:2340-2348.

13. Malbrain ML, Deeren DH - Effect of bladder volume on measured intravesical pressure: a prospective cohort study. Crit Care, 2006;10:R98. 